\title{
Fog and Cloud Computing based Smart Healthcare: A Framework
}

\author{
Suparna Biswas \\ Maulana Abul Kalam Azad University of Technology, WB. \\ Department of Computer Science \& Engg.
}

\begin{abstract}
Current era of smart and green enabling technologies makes smart city and its applications realizable. Smart healthcare is indispensable in a smart city to make citizens' life easy and comfortable. Advancement in internet-of-things, cloud computing, big data, machine learning etc. lead to successful healthcare monitoring and support. Such application demands stringent low latency performance along with other requirements. To facilitate this strict delay limitation specifically in emergency or critical health condition that needs quick response, fog computing can be exploited. Fog computing ensures processing of data closer to its origin thus reducing communication delay and network congestion hence quick feedback in case if an emergency has been identified by processing health data at fog devices. Fog layer does screening of health data based on threshold value or rate at fog layer and if emergency is identified then data is directly sent to doctor/caregiver otherwise data is sent to cloud layer for further processing, storage, offline access etc. An architecture, flowchart and algorithm have been framed to detail working of the proposed framework.
\end{abstract}

\section{General Terms}

Internet-of-things, smart healthcare et.al.

\section{Keywords}

Smart application, end-to-end delay, emergency or critical health condition, sensors, fog, cloud etc.

\section{INTRODUCTION}

With rapid advancement in semiconductor, communication and information technology, Internet of things is a successful technology. And with success of enabling technologies such as IoT, Cloud and Fog Computing, Big data analytics, data mining, artificial intelligence etc., smart city is not a concept, but reality. Smart city is comprised of smart applications such as smart infrastructure, smart transportation, smart healthcare, smart energy etc. Smart healthcare enables citizens to be equipped with an easy, affordable and technology enabled real time solution to lead a quality life. Quality healthcare monitoring and services can be availed irrespective of remoteness of people in rural areas from speciality healthcare establishment generally in urban areas. Moreover, in global perspective, by $2050,75 \%$ of population will live in cities and in India it is already $80 \%$ now. Though, infrastructure and resources growth is not in proportion. Hence available resources, facilities, infrastructures have to be managed intelligently for efficient utilization and availability. In smart healthcare, IoT enabled biophysical sensors, wearable or implantable, monitor biological data with precision and at appropriate interval to send to medical cloud server through heterogeneous communication wired or wireless links for storage, processing, access by doctors/caregivers. Knowledge acquired by processing helps in generation of advice/prescription and in case of any emergency or critical scenario, intelligent control or feedback to be given to the sensing or monitoring unit for intelligent manipulation through control and operating components if required. Now timeliness of advice generation and feedback to be given to people in need in healthcare depends on end-to-end delay of health data from sensing unit to processing unit (maximum permissible delay limit in health data transmission is $250 \mathrm{~ms}$ [1]. If the healthcare monitoring and support is for a person intended for fitness regime or a person in normal health condition, physiological signals sensed can be sent to cloud server for storage [2], getting processed and passing feedback to intended recipients or control system if needed. Delay due to this communication from sensing layer to cloud layer and computation at cloud layer for knowledge extraction and access time by doctor for advice generation may be suitable in normal health condition. So end-to-end delay in data transmission, processing and advice generation has two components - a) communication b) computation. If a person is in need of immediate healthcare support in his abnormal or critical health condition, he can be facilitated with more quick and prompt response by reducing these components wherever possible. Communication delay can be reduced by exploiting successful deployment of fog layer [3] in between sensing layer and cloud layer. Fog computing is a paradigm to manage a distributed environment that can compute and provide network services in between sensing layer and cloud layer, thus making it possible to store and process data closer to its origin. Fog devices e.g. smart gateways, routers, dedicated fog devices etc. have computation ability, memory for storage and situated closer to sensing layer in the network. Moreover, fog computing is suitable in applications that demand low latency such as health care because it supports mobility, computational resources, integration with cloud, heterogenous interface, distributed data analytics etc. Section 2 describes state-of-the art works section 3 presents proposed work with architecture, algorithm flowchart and end-to-end delay analysis finally section 4 concludes the work.

\section{LITERATURE SURVEY}

Several works have been reported in recent times on smart city and its applications [4] [5]. Smart healthcare is indispensable in a smart city. A number of smart healthcare system using health sensors and cloud server has been proposed to provide anytime, anywhere health monitoring and support service to the person in concern [6] [7]. Cloud provides the advantage of customized, demand based processing, storage and offline access ability. Cloud computing ensures processing, storage and retrieval of patients' private information in secure and reliable way [2] [8]. Fog computing is an emerging technology, already being deployed successfully can be exploited in real-time applications that requires low latency performance such as healthcare [9] [10]. Recently healthcare frameworks to enhance performance in terms of latency have been proposed where fog layer works in collaboration with cloud support 
[11]. In this work, fog nodes are entrusted to perform low overhead processing where as big data processing and retrieval of information from big data are accomplished at cloud. Moreover, in association with already existing cloud based healthcare framework, how fog nodes can be placed for efficient low latency performance in theoretically and experimentally demonstrated. Experimental results show latency in fog nodes are less than that in cloud. Simulation of fog based IoT healthcare framework has been done using iFogSim simulator. Following table summarizes some of the relevant literatures reported here:

Table 1: Comparison among related works

\begin{tabular}{|l|l|l|l|l|}
\hline Ref no. & Year & Fog/cloud & application & Implementation \\
\hline$[2]$ & $2-17$ & Cloud & healthcare & Simulation (python) \\
\hline$[6]$ & 2016 & Cloud & healthcare & Framework \\
\hline$[7]$ & 2018 & Cloud & healthcare & Simulation(java) \\
\hline$[8]$ & 2016 & Cloud & healthcare & framework \\
\hline$[9]$ & 2018 & Fog & healthcare & $\begin{array}{l}\text { Sensor based } \\
\text { experiment }\end{array}$ \\
\hline$[10]$ & 2018 & Fog & healthcare & Simulation \\
\hline$[11]$ & 2018 & Fog and cloud & healthcare & ifogSim smulator \\
\hline
\end{tabular}

\subsection{Problem Identification}

In health care domain, sensor based monitoring and support may exploit both body sensors and environmental sensors distributed at different locations. Big health data are generally collected and aggregated by IoT networks and are sent uplink to cloud server for storage and processing. Cloud server supplements the scarcity of infrastructural support pay-per-use basis. But delay and latency caused in transfer of data to cloud and back to the application may always not be suitable in health care domain especially in emergency and critical condition when quick and immediate response is required. Fog computing plays a pivotal role here by facilitating data processing closer to the origin of data i.e. closer to the sensors and brings computing towards the edge network. Thus communication latency can be reduced and network congestion can be avoided. In case of critical or emergency health condition, health data can be sent to doctor from fog layer itself instead of sending to cloud layer for reducing communication delay and to provide quicker response and advice to the person in emergent need of help. Figure 1. [12] shows the delay comparison between cloud and fog computing as a reference.

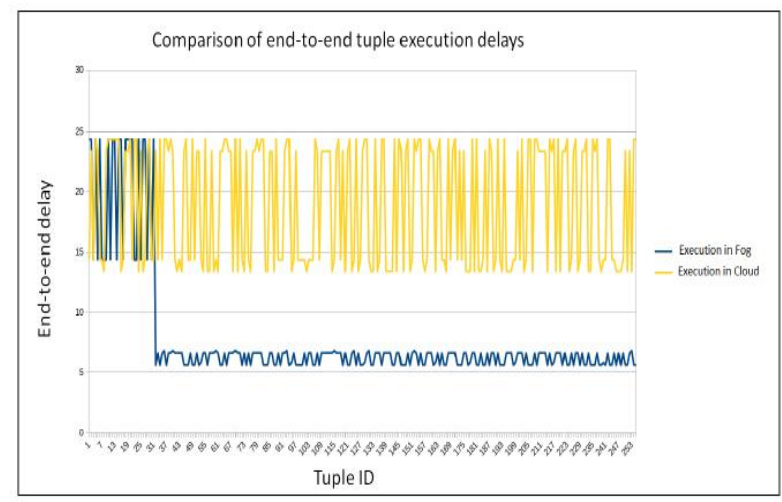

Figure.1 Delay comparison between cloud and fog computing [3]

\section{PROPOSED WORK}

The application demanding low latency requirement considered here is healthcare in which vital physiological signals of the person are sensed by different heterogenous sensors at appropriate intervals for further processing at cloud server. Purpose is to continuously and remotely monitor the person in concern and provide him with proper alert and advice by the caregiver who can remotely and securely access data through cloud anywhere, anytime. Health condition of a person may be stable or may fluctuate abnormally. Thus following scenarios are considered.

Scenario 1: Now in case of normal health condition, this system works satisfactorily as if end-to-end data transmission time falls below a certain delay limit .e. $240 \mathrm{~ms}$ for healthcare. This is feasible as reported in [1].

Scenario 2: In emergency health scenario, such as heart attack or unrest of a heart patient, abrupt sugar or pressure level fluctuation, sudden fall of a critically ill patient and its effect such as stroke etc., abruptly changed vital health data must be processed for knowledge extraction and decision making quickly for immediate support. Moreover, all sensor data may not provide emergency or critical health data at same time. So if a subset of sensor data that are giving abnormal data can be processed more quickly and sent to doctor for his immediate access and advice generation in less time, it would be lifesaving. Here fog devices are exploited to accomplish all processing and decision making in reduced communication time [13]. 


\subsection{Proposed Architecture}

The architecture proposed in figure 2 illustrates above scenarios.

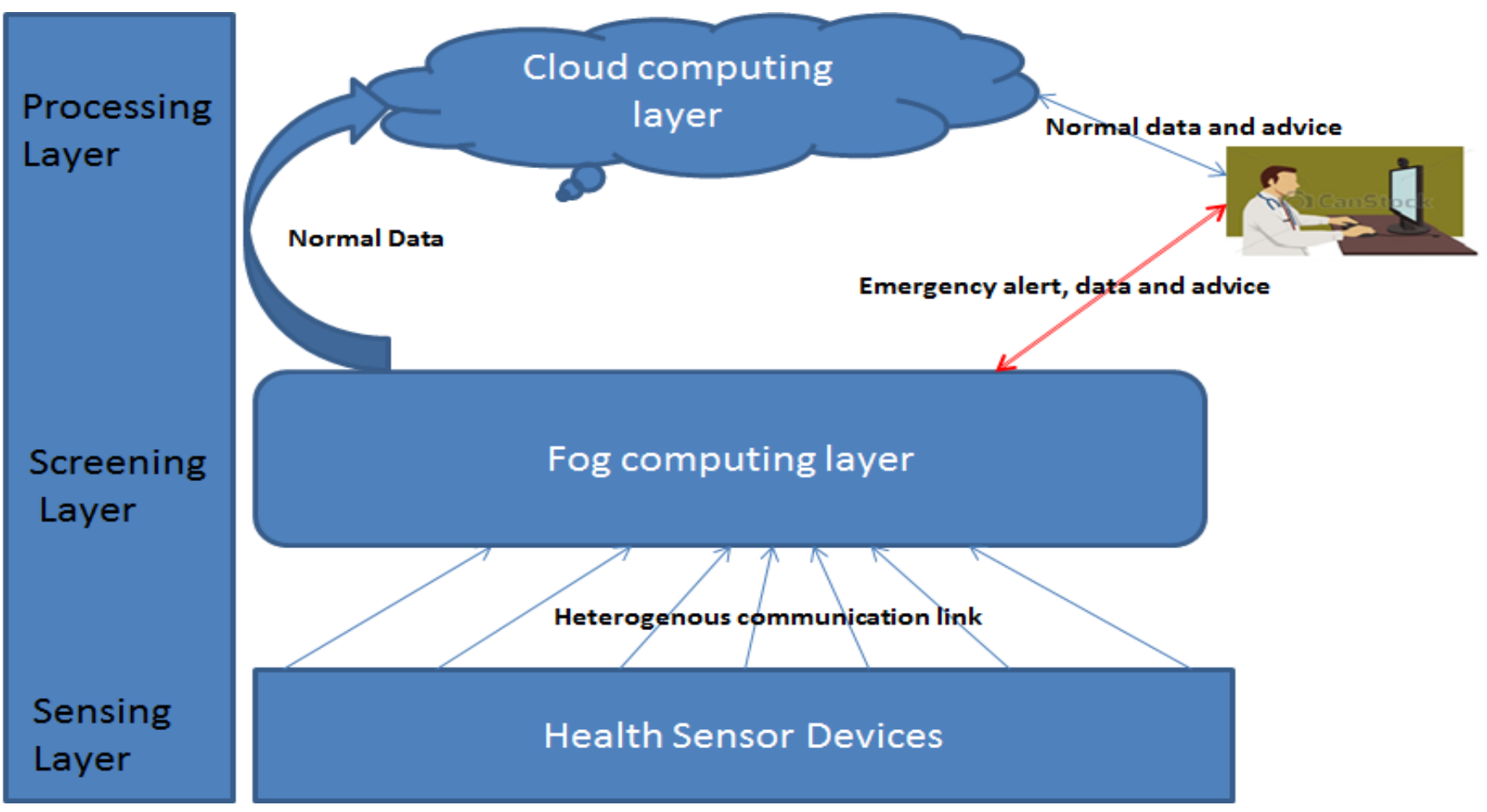

Figure 2. Architecture for healthcare support both in normal and emergency scenario

The three layer architecture comprising of sensing layer, screening layer and processing layer to support both normal and emergency healthcare scenario is depicted in figure 2. In sensing layer there are a number of health sensors deployed on human body or in environment for sensing different vital human and environmental health parameters. Sensors are heterogenous in nature and sense data or signals of different value and rate. These sensors have wireless connectivity with fog computing layer which is the immediate upper layer of sensing layer and cloud computing layer, next upper layer of sensing layer. Sensor may use heterogenous communication links such as, WiFi, Wimax, Zigbee, Bluetooth, IEEE 802.15.4. LAN etc. to send data to upper layers. Sensed data reaches to fog computing layer for screening. Each health parameter has well defined normal value, which may be a single value or a range depending on the particular data type and abnormal value or range. Sometimes some data is expressed in terms of rate e.g. heart rate or pulse rate etc. Using standard benchmark medical database available [9] [14]
[15], screening of sensed data may be done at fog layer to classify the data in normal or abnormal class. If data is found to be normal, it will be forwarded to cloud for further processing, storage and offline access by the doctor or caregiver as per routine or schedule for advice generation or simply continuous monitoring and report generation. If data is found to be in abnormal class then an alert message will be generated and will be delivered to doctor's smartphone using push message option along with health data for immediate advice generation and proper treatment and support needed immediate then. Data will be temporarily saved to fog devices' storage and once screening activities are over, data will be sent to cloud layer for storage and future reference whenever required. Advantage of using fog devices for handling with emergency health data is that fog devices are distributed hence failure can be avoided and processing of data closer to the source of data can be ensured. 


\subsection{Flowchart}

START:

STEP 1: Sense physiological signal Si, I = 1....n; // $n$ is number of sensors

STEP 2: Send Si to fogi, $\mathrm{j}=1 \ldots \mathrm{m} ; / / \mathrm{m}$ is number of fog devices

STEP 3: $\forall ' \mathrm{Si}, \mathrm{i}=1 \ldots \mathrm{n}$,

STEP 3.1: if( $\mathrm{i}>$ Thi) // screening at fog layer, Thi $=$ threshold of signals from 1 to $n$ sensors f

STEP 3.1.1: scenario $=$ = emergency / critical;

STEP 3.1.2: response_type $==$ quick;

STEP 3.1.3: processing_layer $==\mathrm{fog}$;

STEP 3.1.4: Doctors access data from fog layer;

STEP 3.1.5: Alert message sent to patient or relative from fog layer;

\section{STEP 3.2: else}

STEP 3.2.1: scenario $=$ = normal;

STEP 3.2.2: response_time $=\mathbf{=}$ permissible delay in healthcare;

STEP 3.2.3: processing_layer $==$ cloud;

STEP 3.2.4: Doctors access data from cloud layer;

STEP 3.2.5: Alert message sent to patient or relative from cloud layer,

END

Figure 3: Working algorithm describing normal and emergency healthcare support using fog and cloud

The algorithm depicted above details about handling of both normal and emergency health data ensuring low latency

\subsection{Flowchart}

The flowchart in fig. 4 describes the working of the proposed algorithm to classify health data into normal and abnormal class to identify health condition as normal or abnormal that may be mapped to emergency or critical scenario. In normal health condition, health data are stored and processed at cloud layer and as per knowledge extraction, reported to doctor and requirement. Emergency data is not sent to cloud to avoid delay due to communication and network congestion.

doctor accesses data as per predefined schedule or routine. But in case of emergency health scenario, alert message is pushed into doctor's smartphone for prompt access to the health data so that immediate advice and care can be taken at proper time. Delay in such scenario can cause havoc on patients' life. 


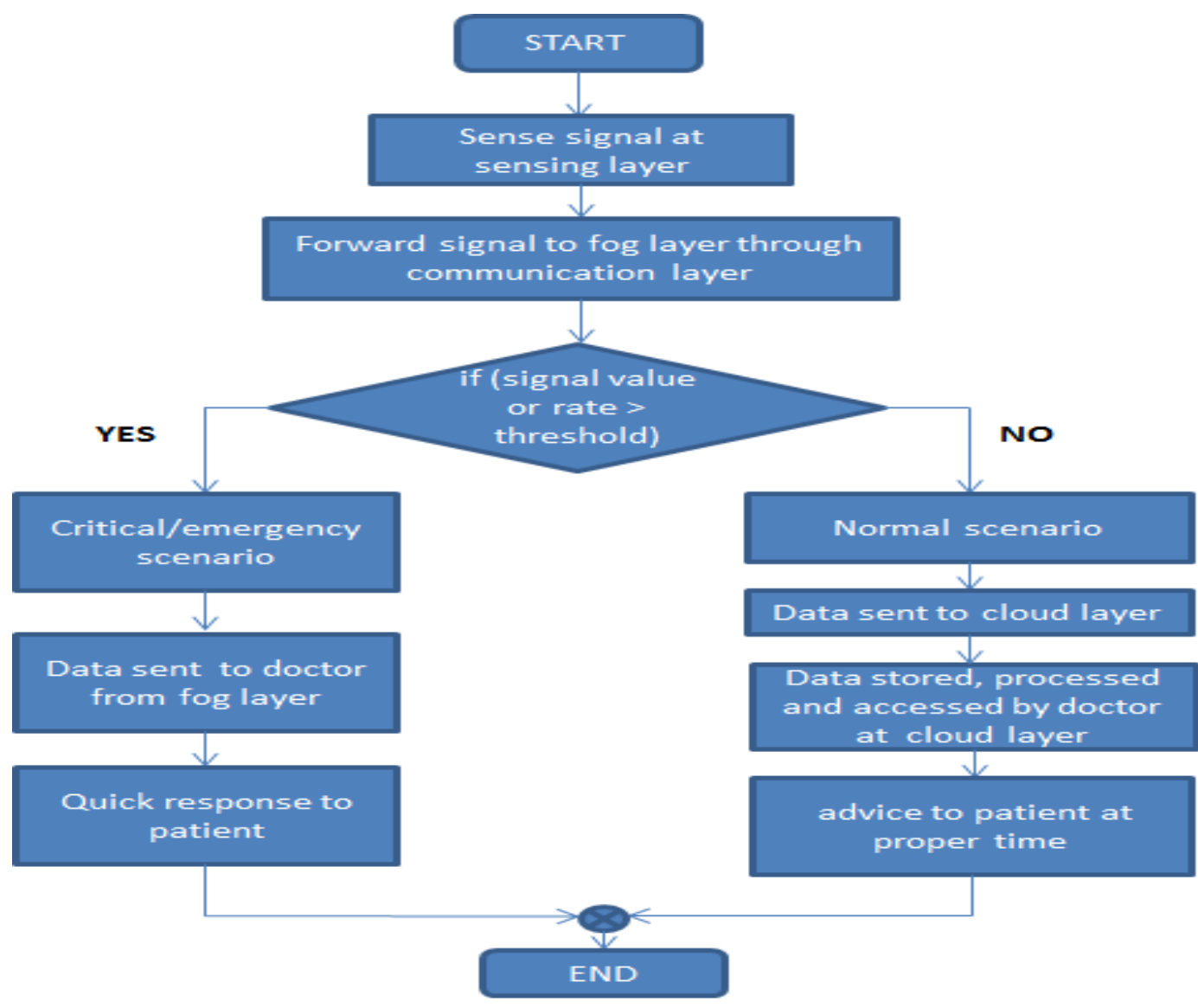

Figure 4. Architecture for healthcare support both in normal and emergency scenario

\subsection{End-to-end-delay analysis in proposed architecture}

End-to-end delay of sensed vital health data is one of the major factors in successful and timely remote health care support whenever necessary. Now as per the architecture shown in figure 2, total time needed to reach health data from sensing layer to doctor or caregiver is termed as end-to-end delay and it has a number of components. This delay in scenario 1 and scenario 2 described above are illustrated further:

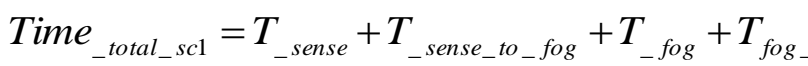

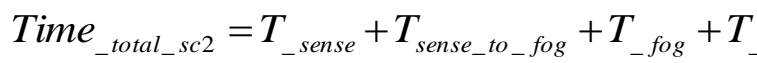
Now, $\quad\left(\right.$ Time _total_sc1 $\left._{-}<T i m e_{\text {_total_sc } 2}\right)==T R U E$, because communication delay in Time total_sc2 $_{-}$is reduced by $T_{\text {fog_to_cloud }}$ time component.

Abbreviations used for different time components are illustrated below:

\section{$T_{-}$sense $=$time to sense data}

$T_{\text {sense_to } f \text { fog }}=$ time to send data from sensing layer to screening layer i.e. fog layer

$T_{-f o g}=$ time for screening data

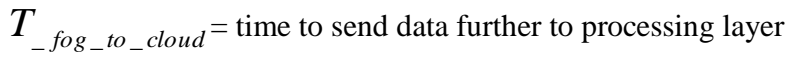

$T_{\text {access }}=$ time to access data from cloud by doctor

\section{CONCLUSION}

Advancement in enabling green smart technologies such as IoT, big data, fog and cloud computing etc., smart city and applications are emerging area of research nowadays. Smart healthcare is indispensable part of it. One of QoS requirement of healthcare application is low latency or end-to-end delay of health data from point of origin to processing and feedback. Several healthcare frameworks based on cloud have been reported since sensors generate big data and cloud can support retsdircess required to store, process and retrieve information on demand basis. But low latency is a great concern, hence exploitation of fog devices having storage, computation and communication capabilities makes reduction in communication delay. But as resources in fog devices are limited, so only to process emergency data faster to provide the person in need quicker response by the doctor, fog devices are entrusted for this. In proposed architecture fog devices work in collaboration with cloud service to realize smart healthcare. Flowchart, algorithm and end-to-end delay analysis give insight to the proposed smart healthcare architecture exploiting fog and cloud.

\section{REFERENCES}

[1] R.Gupta, G.Pradhan, s.Biswas, "Polling vs No Polling: QoS driven performance analysis of IEEE 802.15.6 for varying data rate in WBAN", in proceedings of IEEE ANTS 2017.

[2] Sohail Saif, Rajni Gupta, Suparna Biswas, "Implementation of cloud assisted secure data transmission in WBAN for healthcare monitoring", 
International Conference on Advanced Computational and Communication Paradigms (ICACCP-2017),in proceedings of ICACCP-2017 pp 67.

[3] A.V.Dasterdi, H.Gupta, R.N.Calheiros, S.K.Ghosh, R. Buyya, "Fog Computing: Principles, Architectures, and Applications ", chapter 4,

[4] A. Zanella, N. Bui, A. Castellani, L. Vangelista and M. Zorzi, "Internet of Things for Smart Cities," in IEEE Internet of Things Journal, vol. 1, no. 1, pp. 22-32, Feb. 2014.

[5] J. Lin, W. Yu, N. Zhang, X. Yang, H. Zhang and W. Zhao, "A Survey on Internet of Things: Architecture, Enabling Technologies, Security and Privacy, and Applications," in IEEE Internet of Things Journal, vol. 4, no. 5, pp. 1125-1142, Oct. 2017.

[6] S. Tyagi, A.Agarwal, P.Maheshwari, "A conceptual framework IoT - based healthcare system using cloud computing", 2016 6th International Conference - Cloud System and Big Data Engineering (Confluence).

[7] A.S. Pawade, R.S. Jamgekar, "MediCloud: Cloud Computing Services to Health Sector", International Journal of Computer Applications (0975 - 8887) Volume $179-$ No.23, February 2018

[8] D.Raval, S.Jangale, "Cloud based Information Security and Privacy in Healthcare", International Journal of Computer Applications (0975 -8887) Volume 150 No.4, September 2016.
[9] B. Barahani, "Exploiting smart e-Health gateways at the edge of healthcare Internet-of-Things: A fog computing approach", Future Generation Computer Systems Volume 78, Part 2, January 2018, Pages 659-676.

[10] S.k. Sood, I.Mahajan, "A Fog-Based Healthcare Framework for Chikungunya", IEEE Internet of Things Journal, Volume 5, Issue 2, ISSN: 2327-4662, April 2018.

[11] R.Mahmud, F.Luiz Koch, R.Buyya, "Cloud-Fog Interoperability in IoT-enabled Healthcare Solutions", in proceedings of $19^{\text {th }}$ ICDCN, 2018.

[12] Stantchev, Vladimir, et al. "Smart Items, Fog and Cloud Computing as Enablers of Servitization in Healthcare." Sensors \& Transducers (1726-5479)185.2 (2015).

[13] Cao Yu, et.al., "FAST: A fog computing assisted distributed analytics system to monitor fall for stroke mitigation.", Networking, Architecture and Storage (NAS), 2015 IEEE International Conference on

[14] https://www.urmc.rochester.edu/encyclopedia/content.as px?ContentTypeID $=85 \&$ ContentID $=\mathrm{P} 00866$

[15] S. Flemming et.al., "Normal ranges of heart rate and respiratory rate in children from birth to 18 years: a systematic review of observational studies", PMC, doi: 10.1016/S0140-6736(10)62226-X. 